\title{
Inszenierungen und Bedeutung von Pausen in den Kabaretts von Josef Hader
}

\author{
[The staging and significance of pauses in cabarets of Josef Hader] \\ http://dx.doi.org/10.11606/1982-88372444314
}

Rainer Guggenberger ${ }^{1}$

\begin{abstract}
This article deals with pauses, breaks and silence, and their dramatic functions and meanings, which can be detected within the cabarets of the Austrian comedian Josef Hader. From a general point of view, we reflect on the (anti-)phenomenon of pauses and silence and their essences, searching to define them and taking the pauses in music as starting point and theoretical analogon.
\end{abstract}

Keywords: Josef Hader; Pauses; Silence; Dramatic functions; Austrian cabaret; Music and drama.

Resumo: Este artigo trata de pausas, interrupções e silêncio e as suas funções e os seus significados dramáticos que podem ser detectados nos esquetes teatrais do comediante austríaco Josef Hader. De um ponto de vista geral, nós refletimos sobre o (anti)fenômeno de pausas e silêncio e as suas essências, tentando definí-los, tomando as pausas na música como ponto de partida e análogon teorético.

Palavras-chave: Josef Hader; Pausas; Silêncio; Funções dramáticas; Esquetes teatrais austríacos; Música e drama.

Zusammenfassung: Dieser Artikel behandelt Pausen, Unterbrechungen, Stille und deren dramatische Funktionen und Bedeutungen, die sich in den Kabaretts des österreichischen Komödianten Josef Hader ausmachen lassen. In allgemeiner Weise wird dem (Anti)Phänomen der Pausen und Stille sowie dem ihnen Wesenhaften nachgespürt, um zu einer Definition zu gelangen. Dabei werden die Pausen in der Musik als Ausgangspunkt und theoretisches Analogon herangezogen.

Stichwörter: Josef Hader; Pausen; Stille; Dramatische Funktionen; Österreichische Kabaretts; Musik und Drama.

\section{Einleitung}

Wenn Aristoteles feststellt, dass eine gelungene Tragödie nicht nur als Bühnenspektakel funktioniert, sondern auch als Lesetext (cf. poet. 1462a), so mag er sich gegen eine

\footnotetext{
${ }^{1}$ Universidade Federal do Rio de Janeiro, Faculdade de Letras, Departamento de Letras Clássicas, Av. Horácio Macedo, 2151, Rio de Janeiro, RJ, 21941-901, Brasil. E-mail: rainer@ letras.ufrj.br. ORCID: 00000003-0543-2606
}

\section{(cc) BY-NC}


GUGGENBERGER, R. - Inszenierungen und Bedeutung von Pausen in Josef Hader

(hyper)visuelle Inszenierungspraxis (ő $\psi 1 \varsigma^{2}$ ) wenden, ${ }^{3}$ und insofern die Notwendigkeit des Seherlebnisses als conditio sine qua non für das Zustandekommen einer gelungenen Rezeption des Plots $(\mu \tilde{v} \theta 0 \varsigma)$ bestreiten. ${ }^{4}$ Nicht impliziert ist damit eine gleichzeitige

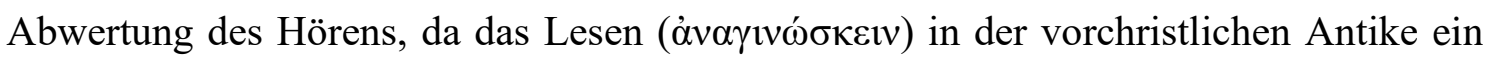
lautlich artikulierter (vielleicht sogar mit Gesten ${ }^{5}$ unterstützer), sozialer Akt war: nicht so sehr ein Vorlesen als vielmehr etwas, das in Richtung eines Vortrages oder einer Deklamation ging, ${ }^{6}$ die im Beisein von interessierten Zuhörern stattfand, welche wohl oft aufmerksamer lauschten und sich auf den Plot mehr einließen, als ein Großteil der athenischen Theaterzuschauer, der wohl die feinen Nuancen von sophokleischen und euripideischen Tragödien intellektuell nicht (oder zumindest nicht immer adäquat) zu fassen vermochte, sondern den Theaterbesuch eher als Gelegenheit wahrnahm, um einem Spektakel beizuwohnen. ${ }^{7}$ Dass Aristoteles an der Stelle poet. 1462a, wo er vom Lesen

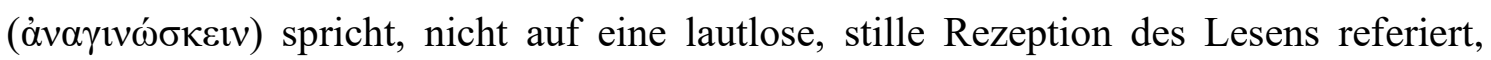
sondern auf eine akustische Rezeption, d.h. von Zuhörern ausgeht, bekräftigt poet. 1453b, wo postuliert wird, dass der tragische Plot so beschaffen sein müsse, dass ohne das Sehen

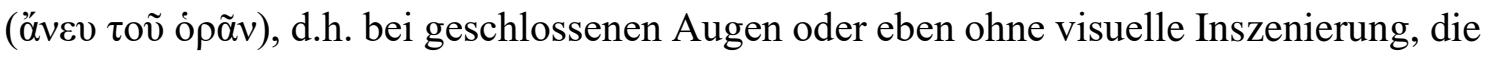
der Tragödie eigentümlichen Affekte evoziert werden: Aristoteles verwendet an dieser

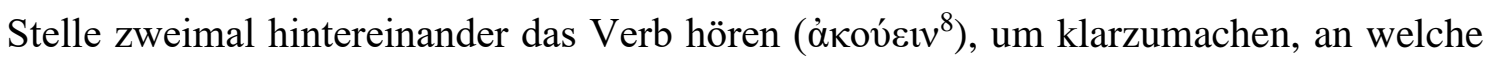
Art von Rezeption er denkt. Was Aristoteles also sagen möchte, ist, dass eine gute Tragödie auch dann ihre plotinhärente bzw. -adäquate Wirkung erzeugt, wenn die Schauspieler $^{9}$ fehlen. Dabei war es sicherlich nicht unwesentlich, wie der Vorleser

\footnotetext{
${ }^{2}$ „Mit Opsis meint Aristoteles allein das Aussehen der Schauspieler und des Chores (d.h. ihre Masken und Kostüme etc.), ihre performativen Bewegungen samt ihrem kanonisierten Gestenrepertoire und eben nicht die vorhandene szenische Kulissendekoration." (MERKER 2016: 140, Anm. 62).

${ }^{3}$ Cf. Guggenberger, 2016, p. 224-225, Anm. 9. Dort Schmitt, 2008, p. 332 und insbesondere Marzullo, 1980, p. 193: „Aristoteles bekämpft [...] eine neue Dramaturgie, die er für willkürlich, ästhetisierend, formalistisch hält. Gegen das bestürzende Überhandnehmen der verschiedenartigen Ausdrucksmittel, denen er nur eine Nebenrolle zugesteht, fordert er letztlich den Primat des ,Wortes'.“.

${ }^{4},[I] \mathrm{n}$ diesem Punkt kann ich mich nicht genug beglückwünschen, mit Aristoteles einer Meinung zu sein, für den die ganze Entwicklung der Theaterkünste auf der Ebene des Hörens geschieht, wobei das Schauspiel für ihn eine Einrichtung am Rande ist. Die Technik ist gewiß nicht nichts, aber sie ist nicht das Wesentliche [...]. Das Schauspiel ist hier ein sekundäres Mittel.“ (LACAN 1996: 304).

5 Übertrieben gestikuliert wurde beim nicht szenischen Vortrag eines antiken Plots aber wohl nicht. Zumindest spricht Aristoteles im Zusammenhang mit dem bloßen Vorlesen einer Tragödie davon, dass die

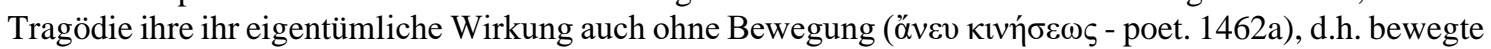
Darstellung, erreiche.

${ }^{6}$ Cf. Guggenberger 2016, p. $227 \mathrm{ff}$.

${ }^{7}$ Mit einem (verhätlnismäßig) leisen und zivilisierten Burgtheaterpublikum hatte es wenig gemein. Berichte über mobile Getränke- und Essensverkäufer - ähnlich jenen in Fußballstadien - sind uns überliefert.

${ }^{8}$ Beidemale in Form des präsentischen Partizips.

${ }^{9}$ Zumindest in ihrer eigentlichen Funktion als Schauspieler - denn freilich mag es Schauspieler gegeben haben, die abseits der Aufführungen und ohne Bühne Dramentexte vortrugen.
} 
GUGGENBERGER, R. - Inszenierungen und Bedeutung von Pausen in Josef Hader

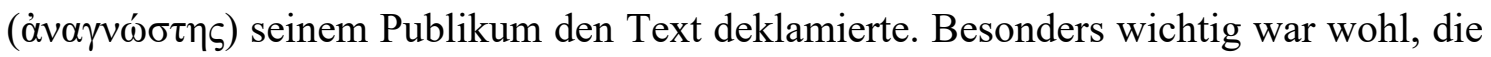
Pausen richtig zu setzen bzw. zu inszenieren ${ }^{10}$.

Es mag müßig sein, zu untersuchen, ob die Kabaretts von Josef Hader sich in die Reihe derjenigen literarischen Stïcke einordnen lassen, die das aristotelische Postulat, das ja eigentlich auf eine gelungene altgriechische Tragödie (und also nicht auf modernes Theater und Kabarett ${ }^{11}$ ) abzielte, erfüllen. Der Beitrag möchte jedoch aufzeigen, dass Haders Kabaretts sich zwar insofern mit modernem dramatischem Theater berühren, als Hader sich theatraler Mittel bedient und seine performances theatralisiert, darüber hinaus jedoch - anders, bzw. zumindest besser als dramatisches Theater - auch als Hörtext bestens funktioniert. Dabei ist wiederum interessant, dass Hader umgekehrt als Lesetext nicht funktioniert, dramatische Theaterplots mitunter (wenn auch wohl meist mit Abstrichen) jedoch schon. ${ }^{12}$ Hader selbst widerspricht jedenfalls explizit einer möglichen Loslösung seiner Kabaretts vom Akt des erzählerischen Darstellens, wenn er feststellt:

Man verändert auch immer wieder bei der Überarbeitung das, wie es klingt. Drum hab“ ich dann auch Schwierigkeit ein Buch herauszugeben [d. h. den Kabaretttext als gedruckten Text herauszugeben]. Weil das eine reine Partitur für Klang ist - das ist kein Text zum Lesen. (ARENDS 2011: 119).

\section{Zur Deutung der Kabaretts von Josef Hader als „reine Partitur}

\section{für Klang"}

Die vorliegende Arbeit versucht dem Problemstand entgegenzuwirken, dass das Akustische, als das Gehörte bzw. - im Fall der Pausen - das Nichtgehörte, ${ }^{13}$ in Kabaretts seitens der Musikwissenschaft nicht behandelt wird, da es sich dabei (überwiegend) nicht

\footnotetext{
${ }^{10}$ Auch wenn es hier - etwas wider den Wortsinn - um eine Inszenierung geht, die eben abseits der Bühne

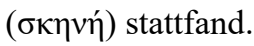

${ }^{11}$ Interessant ist, dass Aristoteles sich sowohl in poet. 1462a als auch in $1453 \mathrm{~b}$ auf die Tragödie bezieht und zudem als Beispiel den Ödipus-Mythos nennt, in welchem für den tragischen Held das Visuelle dermaßen unerträglich wird, dass er sich, in die Augen stechend, der Sehkraft beraubt; jene Rezeptionsform die Ödipus bleibt ist also eben das Hören. Es scheint folglich, dass sich Aristoteles' Behauptung, dass ein guter Plot auch ohne Inszenierung funktioniere, ausschließlich auf die Tragödie bezieht, und eben nicht auch auf die dem Kabarett eher verwandte Komödie. Das kann bedeuten, dass Aristoteles es nicht für möglich hielt, dass auch die (attische) Komödie als „bloßer“ Vortrag funktioniert hätte - da brauchte es eben dann doch der theatralen, rustikal-derben, dafür urkomischen Aufführung durch geeignete Schauspieler.

12 Besonders Ende des 18. und im 19. Jahrhundert finden sich im deutschen Sprachraum dafür zahlreiche Beispiele: Schiller, Grillparzer etc.

${ }^{13}$ Es werden also nur die der Kommunikation inhärenten Dimensionen des Akustischen, also - im

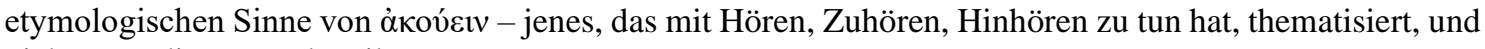
nicht etwa die Raumakustik.
} 
GUGGENBERGER, R. - Inszenierungen und Bedeutung von Pausen in Josef Hader

um Musik handelt, und dass es andererseits in der Theaterwissenschaft vernachlässigt wird, zugunsten einer (Über)Betonung des Visuellen in theatralen Formen. Zugleich sollte auch ein 50 Jahre altes Desiderat der Musikwissenschaftlerin Zofia Lissa erfüllt werden, das darin bestand, dass doch jemand auch ,die Wirkungsformen der Stille in verschiedenen zeitlichen Künsten“" (LISSA 1962: 346) abseits der Musik untersuchen möge.

Die Beschränkung auf die rein akustische Dimension der Performanz Haders erlaubt, nicht-akustische Kommunikationsformen und Interaktionsmanifestationen auszuklammern und sich auf die Pause als akustisches Erlebnis und akustischen Ausdruck zu konzentrieren. Die emotional eindringlichsten Pausen Haders sind die „stillen“ und „offenen Pausen“14, die im Drucktext nicht wirken können, sondern der Aufführung insbesondere der Stimme als Medium, das selbst nicht textlich ist, den Text jedoch hervorzubringen hilft - bedürfen, obgleich sie einmal aufgeführt auch rein-akustisch, also abgelöst vom audiovisuellen Ereignis, wirken können. Es zeigte sich, dass für ein Verständnis und eine Erklärung der Kabaretts Josef Haders über weiteste Strecken die rein-akustische Wahrnehmung hinreicht, obgleich es einzelne Passagen gibt, deren semantischer Ausdruck rein-akustisch - bspw. im Radio - nicht adäquat wahrgenommen werden kann, wozu etwa das einzelne Stillen begleitende (oder gar sie evozierende) Lächeln gehört ${ }^{15}$. Inwieweit durch die fehlende visuelle Ebene die gesamtkünstlerischen Ausdrücke in den Kabaretts Haders nicht adäquat vermittelt werden können, wurde in der vorliegenden Arbeit nicht thematisiert. Für die Arbeit an diesem Artikel wurde außerdem bewusst nicht die Methodik von Theoretikern von Körper-Inszenierungen übernommen, eine Methodik die, wo sie die Funktions- und Wirkweise von Theater und Kabarett ergründen will, dies in der Regel ausschließlich vom Standpunkt des Präsenzpublikums und den momentan körperlich anwesenden KünstlerInnen aus versucht. Dabei besteht die latente Gefahr bei Beschreibungen von Vorgängen und der Formulierung von Theorien bestimmte Erscheinungen fälschlicherweise körperlich-visuellem Darstellen zuzuschreiben, da verabsäumt wird gleichberechtigt mit den visuellen Phänomenen auch das (körperlich-)akustische Darstellen bzw. die akustischen Darstellungen auszuwerten.

\footnotetext{
${ }^{14}$ „An open silence is one whose precise meaning and effects, because they cannot be determined by analysis of the words of the playtext, must be established by nonverbal, extratextual features of the play that emerge only in performance" (MCGUIRE 1985: xv; nach JAWORSKI 1993: 94).

${ }^{15}$ Bspw. Minute 1.44 bis 1.47 auf der DVD Josef Hader. Privat. Wien: Hoanzl, 2008 (aufgezeichnet in der Burgarena Finkenstein 1996), die den ganzen Artikel hindurch als Referenzaufnahme herangezogen werden wird.
} 
GUGGENBERGER, R. - Inszenierungen und Bedeutung von Pausen in Josef Hader

Durch die bewusste Überbetonung der akustischen Dimension von Kabaretts in dieser Arbeit, sollte auch darauf hingewiesen werden, dass es von wissenschaftlichmethodischem Vorteil wäre, nicht nur die Wirkung künstlerischer Vorgänge auf ein Präsenzpublikum zu evaluieren, sondern diese aus mehreren Perspektiven auszuwerten: sich also anzusehen und anzuhören, wie derselbe Vorgang im selben Stück auf verschiedene Arten von Medienkonsumenten wirkt (Bühne, Radio, Fernsehen, CD, DVD) und wie durch die verschiedenen Medien die Funktions- und Wirkweisen desselben Theater-, Kabarettstücks etc. modifiziert werden.

Ausgehend von einer audiovisuellen Analyse der Kabaretts Josef Haders konnte festgestellt werden, dass bei Hader nicht so sehr der Text zum körperlichen Schauspiel, sondern vielmehr das körperliche Schauspiel zum Text als akustischem Event passen muss. Die Kabaretts Haders stehen der Sprache, also dem akustischen Ausdruck ungleich näher als dem körperlich-visuell-nicht-akustischen, dem Drama viel näher als der Pantomime. Wenn bspw. Kabarettbesucher die Performanz Haders im Freundeskreis aufgreifen oder imitieren, tun sie das sprachlich, ohne zusätzlich körperlich-darstellende Eigenarten Haders zu imitieren. Damit soll jedoch nicht geleugnet sein, dass die körperliche Präsenz Haders dem Akustischen eine weitere und breitere Dimension verleiht. Diese Dimension ist aber nicht essentiell für das Gelingen der Kabaretts, auch wenn Haders Schauen, bspw., noch so charakteristisch und gekonnt ist, und obwohl sich allgemein in Pausen und Momenten der Stille innerhalb von Bühnenwerken

visuell erfaßbare Gesten realisieren [können], welche diese [Pause oder] Stille mit einer Aussage füllen, die in ihrem Ausdruck sogleich beredter und zugleich feiner sein kann als Worte. [...] Stille voller Handlung, Gedanken, Gefühle und Spannung. (LISSA 1962: 317).

\section{Pausen und Stille als theatrales Mittel Josef Haders}

Bezüglich der Pausen als theatralem Mittel Josef Haders, soll in diesem Artikel weder über die (provozierten) Pausen und Stillen des Publikums ${ }^{16}$, noch über die Pausen zwischen den Akten, und auch nicht über jene zwischen den Szenen, sondern über die Pausen innerhalb der Szenen gesprochen werden.

\footnotetext{
16 „Man bekommt jede Pause, jede Stille mit. Auch ob dies nun eine Stille der Langeweile ist, oder eine Stille der totalen Angespanntheit“ (KÜCHLER 1995: 245; Hader im Interview).
} 
GUGGENBERGER, R. - Inszenierungen und Bedeutung von Pausen in Josef Hader

Viele tausend „Stillen“ finden sich in Haders Kabaretts. In der ersten Szene ${ }^{17}$ von Privat, in der sich die Figur Josef Hader vorstellt und Ereignisse rund um seine Herkunft und Geburt erzählt, sind innerhalb von 4 Minuten und 37 Sekunden 1 Minute und 38 Sekunden Pausen wahrzunehmen. Das entspricht einem Anteil von 35\% Pausen gemessen an der Aufführungsdauer der ersten Szene. In der für die vorliegende Untersuchung ausgewählten Hauptepisode, in der Hader sein Treffen mit dem Kaiser von Afrika dramatisch erzählt, liegt der Anteil immerhin bei $23 \%{ }^{18}$ - In diesem speziellen Fall hat sogar die auf den Abschluss der Szene folgende Aktpause eine dramaturgisch wichtige und den intraszenischen Pausen vergleichbare Bedeutung: Hader gesteht durch sie „dem Zuschauer eine Verschnaufpause zu, damit sich die Wirkung der vorangegangenen Nummern, vor allem der letzten zwei, ,Das große Puff von Nairobi“ und ,Beim Kaiser von Afrika“ voll entfalten kann.“ (RINGHOFER 2000: 83) - Ob Hader sich in der Verwendung seiner „Stillen“ von Ödön von Horváths dramatischen Stücken inspirieren hat lassen oder nicht, wird nicht Gegenstand dieser Untersuchung sein, Analogien zwischen der Wirkweise der Stillen Horváths und den Pausen Haders lassen sich jedoch, auch ohne profunde wissenschaftliche Vergleiche anzustellen, finden. ${ }^{19}$ In der Episode mit dem Kaiser von Afrika erzählt Hader unter anderem von einem anonymen, heterosexuellen Liebespaar. ${ }^{20}$ Die weibliche Figur flüstert der männlichen zweimalig ein intimes, ,ich liebe dich“ zu, wobei Hader vermittels zweier unterschiedlich gewichteten Pausen ${ }^{21}$ eine knisternde Atmosphäre kreiert. ${ }^{22}$ Der „süße Lärm“ der Liebenden geht in diesen zwei Pausen auf und die Liebesworte erhalten erst in dieser Situation sprachlicher Absenz ${ }^{23}$ ihre wahre, intime, kunstvoll vermittelte Bedeutung. Ton und Bild erstarren in diesen Momenten ${ }^{24}$ höchster und zugleich reduziertester und leisester Dramatik. Hader unterbricht seine „dramatische Narration“, um sie, die sprachliche Unterbrechung hindurch, ihrer szenisch-akustischen Kulmination

\footnotetext{
${ }^{17}$ Minute 0.55 bis 5.32 auf der DVD Privat.

181 Minute 25 Sekunden Pausen von Minute $1 \mathrm{~h} 12.40$ bis $1 \mathrm{~h} 18.47$.

${ }^{19}$ Wann es sich (schon) um Stille handelt und nicht (mehr) um eine Pause, wird im Zuge dieser Arbeit kaum erörtert. Grob kann jedoch resümmiert werden, dass Pause Pause von etwas ist, d.h. in einer Pause ist es nicht notwendig still. Was die Stille anbelangt, kann man wiederum zwischen Stille und absoluter Stille unterscheiden. In der nicht absoluten Stille kann (nämlich) durchaus ein Geräusch (wenn auch auf vergleichsweise niederem Pegel) vorhanden sein.

${ }^{20} 20$ Sekunden Pausen von Minute $1 \mathrm{~h} 15.27$ bis $1 \mathrm{~h} 16.31=31 \%$.

${ }^{21}$ Die zweite der Pausen ist eines der raren Beispiele in Kabaretts, wo eine Pause ,as a discharging factor, facilitating the transition of the sound fabric into silence" (DANIELEWICZ 1998: 104), dient.

2210 Sekunden Pause von Minute $1 \mathrm{~h} 16.17$ bis $1 \mathrm{~h} 16.31=71 \%$.

${ }^{23}$ „Es ist etwas nicht anwesend, wenn es still ist.“ (MEISTER 2009: 228).

${ }^{24}$ ",Jene auf Augenblicke komprimierte Zeit in vollkommenem Stillstand bei zugleich höchster emotionaler Spannung oder auch deren Gegenteil.“ (MEISTER 2009: 227).
} 
GUGGENBERGER, R. - Inszenierungen und Bedeutung von Pausen in Josef Hader

zuzuführen. ${ }^{25}$ Warum solche Unterbrechungen des „dramatischen Flusses“ Bedeutung haben und essentiell für das Gelingen dramatischer Kunst sein können, ist neben der Analyse der unterschiedlichen Funktionen von Pause einer der Hauptuntersuchungsgegenstände der vorliegenden Arbeit. Wie bei Horváth die Stillen, so sind in Haders Kabaretts bestimmte Pausen ,energetische Kraftfelder der Stille“, ein „Meta-Raum von Theatralität“ und „ein Dynamo, an dem sich das Gesellschaftliche erst so richtig auflädt": „das Verstummen selber ist vielfältig und ist Mitteilung.“ Die Pause „gehört zur Sprache, ist ihr eigen, ist der auditiven Dimension der Sprache immanent.“ (MEISTER 2009: 229).

Die Pausen bei Hader sind nicht einfach Sekunden, wo nichts passiert. Den Pausen wohnt eine Kreativität inne. Sie schaffen insbesondere Dramatik und Spannung, wie die zitierte ,ich liebe dich“-Szene zeigt. Das Paradoxon, das der Pause inhäriert, ist, dass im Zeitpunkt der Pause nach außen hin nichts positivistisch Fassbares passiert, die Pause aber auf das Publikum wirkt. Das Faktum, dass die „stille Pause“ aus einem Quantum Stille besteht, bedeutet

nicht das $\mathrm{F}$ e $\mathrm{h} 1 \mathrm{e} \mathrm{n}$ der Musik [oder des theatralen Spiels] oder anderer Laute und auch keine Stagnation der dramatischen Entwicklung. Sie ist [...] ein bewußtes und beabsichtigtes Ausdrucksmittel. In ihr tritt eine Spannung der Emotion in Erscheinung, die musikalische Mittel [bzw. sprachliche im Fall Hader] allein nicht mehr ausdrücken können. Die Stille wird hier also zum Ausdrucksmittel ganz bestimmter dramatischer Inhalte. [...] die Stille [ist] [...] nicht nur ein Fehlen der Klangmaterie. Sie kann mannigfaltige Bedeutung haben und im ästhetischen Erleben des Werkes sowie seiner Konstruktion verschiedene Funktionen erfüllen. (LISSA 1962: 316).

Die Pause ist also „nicht nur klangliche Leere“, sondern kann eine „energetische Erscheinung“ (LISSA 1962: 316) sein. Mehr noch, können Pausen als Ausdruck für Unsagbares $^{26}$ (für dasjenige jenseits der Grenzen der Sprache ${ }^{27}$ ) stehen, genauso wie Fermaten in der Musik bspw. „,ür unaussprechliche Gedanken“ (ELZENHEIMER 2005: 75)

\footnotetext{
25 „Nun gilt es festzuhalten, daß Horváths [wie im vorliegenden Fall auch Haders] ,Stille“ nicht im Gestus einer Sprachlosigkeit aufgeht, sondern mehr intendiert, als mit dem Begriff der ,Sprachlosigkeit‘ erfasst ist. Stille ist bei Horváth [wie bei Hader] auch Motor, Weiterentwicklung.“ (MEISTER 2009: 228).

26 "the extreme psychological states such as madness, ecstasy or outrage are filled by silence when words have failed to provide adequate expression for them” (DANIELEWICZ 1998: 131), ,some real feelings can only be expressed in silence" (DANIELEWICZ 1998: 77).

${ }^{27}$ Wie überhaupt bestimmte nicht phonetische „Sprechakte“ und insbesondere Musik einen wichtigen Bereich des nicht Sagbaren innerhalb der menschlichen Kommunikation abdecken: „music, like the nonverbal stretches of , verbal' communication, lacks descriptive power associated with words. This, however, does not diminish its strength. On the contrary, as Debussy says ,beginning where verbal expression is powerless, music is made for what is inexpressible" (DANIELEWICZ 1998: 196). „Die Figuren der Pause, des Schweigens und der Stille [...] ermöglichen [...] das ,Sichtbarwerden, ,Zutagetreten' der Sprache selbst ${ }^{\star}$ - jenseits ihrer ,Darstellungs- und Mitteilungsfunktion'.“(ELZENHEIMER 2008: 20).
} 
GUGGENBERGER, R. - Inszenierungen und Bedeutung von Pausen in Josef Hader

- wohingegen fermatierte Pausen die Wichtigkeit einer Stelle unterstreichen können. ${ }^{28}$ „Analogously, silence may sometimes be interpreted as the continuation of words, i.e. the only suitable way to reply“ (DANIELEWICZ 1998: 207).

Rests and pauses, however, may also be of obstructive nature, when stopping the movement of sounds, and, consequently, halting the formative process. Instead of producing a continuity effect, they might result in a rhythmic stiffness (gapping or staggering effect). (DANIELEWICZ 1998: 208).

Selbst solche hemmende Pausen sind jedoch nicht mit einem „Nicht-spielen“ gleichzusetzen. Auch in solchen Pausen kann das Spiel Haders größte Spannung und Brisanz aufweisen. Die Pausen im Spiel Josef Haders sind von ähnlich tragender Bedeutung wie die Pausen in gelungen dargebotener Musik. Pausen lassen das Nachfolgende oder Vorangehende in der Regel deutlicher, bedeutender und/oder wuchtiger oder auch intimer erscheinen. ${ }^{29}$ In ihrem geschichtlichen Abriss von der Funktion der Pausen in der Musik konstatiert Danielewicz:

In Baroque, rests started to be used as a suspense medium, intensifying the feeling of expectancy preceding the musical passage to come. This function, often used by Bach, can be compared to the rhetorical means found in different sorts of speeches, as well as in stage performances (drama). [...] the rests are connected with the sequential growing [...] and help to achieve tension (DANIELEWICZ 1998: 98).

Hader arbeitet laut eigenen Angaben nicht wie der Kabarettist Gunkl mit dem Kopf der Leute, sondern mit deren Emotionen. Hader sieht sich auch selbst nicht als „Kopfmensch“. ${ }^{30}$ Es geht ihm nicht um eine Pointe nach der anderen, sondern um einen Spannungsaufbau vermittels Gefühlen und Emotionen, um das Hervorrufen von Emotionen und die Vermittlung der jeweils präsentierten Geschichte im Kontext dieser Emotionen. ${ }^{31}$ Pausen können dabei bspw. eine „kritische Situation“ und eine daraus resultierende Unsicherheit schaffen, was wiederum dem Spannungsaufbau zu Gute kommt. $^{32}$

Menschen, die ein bisschen unsicher sind und sich ein bisschen mehr hinterfragen, denken sich, Vielleicht hat der andere auch recht, überlegen sich $N a$, scheiße, man muss den Standpunkt vielleicht auch sehen und ich hab" mich nie als eine Insel gesehen, sondern

\footnotetext{
28 „Die Verlängerung der Pausen durch die Fermate unterstreicht deren besonders wichtige Ausdrucksrolle in der gegebenen Phase des Werks.“ (LISSA 1962: 346) Cf. die fermatierte Pause nach der zitierten ,ich liebe dich"-Stelle in Privat.

${ }^{29}$ Pause bedeutet: Da kommt noch was. Pause und Stille sind nicht Nichts. Man kann in sie hineinhören, und sie können mitunter mehr enthalten, mehr provozieren, als das Nicht-stille und als die Nicht-Pause.

${ }^{30}$ „Es hat jedenfalls bei mir immer mit Instinkt zu tun, mit Drama, mit Gefühl, mit Emotionen und nicht so stark mit Reflexion.“( ARENDS 2011: 120; Hader im Interview).

${ }^{31}$ „Ich bin [...] sehr besorgt um meine Dramaturgie, daß alles Bewegung hat, daß eine Spannung entsteht.“ (KÜCHLER 1995: A 123; Hader im Interview)

${ }^{32}$ „Spannung entsteht [...] oft durch Unsicherheit, die man dem Zuschauer gibt.“ (ARENDS 2011: 114; Hader im Interview).
} 
GUGGENBERGER, R. - Inszenierungen und Bedeutung von Pausen in Josef Hader

ich war immer unsicher, ob nicht der andere auch recht hat. Es war mir nicht recht, dass er recht hat, ich war kein guter Mensch deswegen, sondern ich hab' gefürchtet, dass der oder die andere auch recht haben, und hab dadurch, glaub' ich, von Anfang an im täglichen Leben immer eingeübt, mich in andere hineinzuversetzen. Ich bin dann aber drauf gekommen, dass das sehr günstig ist, taktisch für einen selber. Weil wenn man sich in den Standpunkt des anderen versetzt, dann kann man besser manipulieren. Ich kann ja auch die Kabarettzuschauer manipulieren, weil ich mich immer in deren Standpunkt versetze. Ich denk' mir immer, wenn ich schreibe, Wie geht's dem Zuschauer grad, was macht der Zuschauer jetzt? [...] ich kann dadurch viel stärker berechnen, wie spannend etwas grad ist, und dann brauch ' ich vielleicht einen Witz nicht, weil ich mir denk', die kleben eh grad in den Sesseln. Das ist der Vorteil, den man [...] gegenüber einem normalen Drehbuchautor hat, dass man als Kabarettist immer ein bisschen abschätzen kann, wie das Publikum reagiert. Nie ganz sicher, aber ein bisschen schon. (ARENDS 2011: 122; Hader im Interview).

Der Funktion von Pausen als Gefühlsverstärker bedient sich Hader bspw. in Im Keller, wenn er vom nachteiligen Kostennutzenverhältnis vom Kinderhaben sinniert und schließlich sentimental wird, wenn er sich vorstellt, dass Kinder zu Weihnachten doch eine Bereicherung wären. Die Pause danach spannt alle Sinne der Zuschauer an, hin auf die erwartete Kulmination, eine vermutete Steigerung der Melancholie, wohingegen Hader mit einer trockenen Pointe ins umso heftigere Lachen entlässt ${ }^{33}$; um danach wieder zum melancholischen Grundton zurück zu driften. Die Lacher an dieser Stelle sind in dem Maße intensiver als andere Lacher, in dem die Pause zuvor die Gefühle und Erwartungshaltung im Vergleich zu anderen Szenen verstärkte; die Rezeptionshaltung des Publikums forciert wurde, indem die Pause die „Gefühlsnerven“ reizte ${ }^{34}$. Haders performances an solchen Stellen konstituieren sich aus der Dialektik von (wiederholter) Spannung und Entspannung. Außerdem bedient sich Hader an besagter Stelle zugleich einer weiteren Funktion von Pausen, nämlich der Kontrastierungsfunktion. ${ }^{35}$ - „Es braucht den Kontrast.“ (KÜCHLER 1995: A 128; Hader im Interview). „Die Erwartung verstärkt durch die Pause die Überraschung, die dem ästhetischen Effekt des Kontrastes immer zugrunde liegt.“(LISSA 1962: 330).

Die Pausen im Umfeld der besagten Stelle sind zugleich psychologischen Stillen äquivalent, die ,emphasises the character's feelings and experience. It is either followed by a decision to verbalise thoughts (outburst of emotions) or to withdraw altogether. [...] it could, for example, be caused by suffering, pride, submission, shame, or fear." (DANIELEWICZ 1998: 156). Schauspieler oder Erzähler wollen dadurch ,,anxiety on the

\footnotetext{
${ }^{33}$ Minute 42.36 bis 42.58 auf der DVD Josef Hader. Im Keller. Wien: Hoanzl, 2008 (aufgezeichnet im Orpheum Graz 1994), die den ganzen Artikel hindurch als Referenzaufnahme herangezogen werden wird. 34 ,tensionstimulating function“ (DANIELEWICZ 1998: 99).

35 „Pauses in speech [...] are [...] often used to emphasize contrast and reinforce the effect of surprise realised by unexpected structures or utterances.“" (DANIELEWICZ 1998: 99).
} 
GUGGENBERGER, R. - Inszenierungen und Bedeutung von Pausen in Josef Hader

stage“ darstellen „,and interest on the part of the audience“ (DANIELEWICZ 1998: 156) fördern.

Bei Hader sind solche und auch andere Formen ${ }^{36}$ von Pausen essentiell für das Gelingen der Gesamtaufführung. Die richtigen Pausen und die richtigen Pausenlängen zu finden, gehört mit zum Schwierigsten bei der theatralen Ausarbeitung und Aufführung eines Kabaretts. Eine zu lange musikalische oder dramatische Stille, bspw., verursacht erwiesenermaßen eine Überspannung und Unruhe im Publikum. (Cf. LISSA 1962: 325) Analysiert man bspw. Haders verschieden geformte Pausen, die an der unterschiedlichen Sprechflussführung und den anderen Wortbetonungen innerhalb derselben Geschichte und Pointe in Im Keller einerseits und der Sendung Aschermittwoch der Kabarettisten 2009 andererseits mitverantwortlich sind, so lässt sich erklären, warum Hader einmal mehr Aufmerksamkeit und Gelächter erntet ${ }^{37}$ und einmal weniger ${ }^{38}$. Die Beschaffenheit von - im konkreten Fall emphatischen ${ }^{39}$ - Pausen, Wort- und Satzbetonungen sowie die konkreten Formulierungen und Artikulationen sind, wie dort unschwer ersichtlich, ein Drahtseilakt und entscheiden maßgeblich über den Erfolg einer Aufführung.

Desweitern kann, was gesagt wird, mitunter erst korrekt gedeutet werden, wenn die Pausen, die das Gesagte umgeben, richtig interpretiert wurden. Daneben sind Pausen insbesondere auch notwendig, um strukturiert zu sprechen, d. h. um sich verständlich machen zu können. Tempoexzesse ohne Pausen sind nur ausnahmsweise ${ }^{40}$ als stilistisches Mittel rezipierbar, aber auf Dauer überfordernd. Pausen in ihren mannigfachen Formen sind in der alltäglichen und theatralen Praxis also conditio sine qua non glückender Interaktion.

[A]lthough the significance of silence can usually be interpreted only in relation to sound, the reverse may also be the case, with the significante of sound depending on the interpretation of silence. Silence that constitutes part of the linguistic or musical discourse is more than mere absence of sound. [...] Silence may be considered as a frame in which talk acquires meaning, or as the nonverbal activity which structures interaction (DANIELEWICZ 1998: 198-199).

\footnotetext{
${ }^{36}$ Die dann bspw. im Gegenteil dem „discharge of tension“ dienen, also als „relief medium“ (DANIELEWICZ 1998: 99) fungieren.

${ }^{37}$ Minute 19.08 bis 20.24 und 24.01 bis 24.50 auf der DVD Im Keller.

${ }^{38}$ Cf. Josef Hader beim Aschermittwoch der Kabarettisten 2009, wo manche der Pointen von Im Keller zu gehetzt wirken.

${ }^{39}$ „Emphatic' pauses and rests also belong to the set of stylistic devices; they make the listener, for instance, pay close attention to what is being said or played. They may be of contrastive nature, when preceding something unexpected, surprising, shocking, or, generally, signalling a change." (DANIELEWICZ 1998: 205).

${ }^{40}$ Hader redet sich nur ganz selten in einen „Temposog“: bspw. von Minute 40.55 bis 41.05 auf der DVD Im Keller.
} 
GUGGENBERGER, R. - Inszenierungen und Bedeutung von Pausen in Josef Hader

Von besonderer Bedeutung in den Kabaretts Haders ist eine bestimmte Form der „formulaic silence“(DANIELEWICZ 1998: 199), nämlich jene, die von einem aufgesetzten, resignierend wirkenden Lächeln begleitet ist. ${ }^{41}$ Diese Art von Pause ist ohne Bildunterstützung schwer korrekt perzipierbar, und schon gar nicht antizipierbar. Eine „,interaction structured through silence‘, where the silent signals override speech in the interpretation of the communicative behaviour“ im Gegensatz zu einer „interaction structured through talk“ (DANIELEWICZ 1998: 199) findet sich paradigmatisch im Film Cappuccino Melange, wo Hader den silence-part und Enrica Maria Modugno den talkpart verkörpern.

Mitunter können Pausen so wichtig werden, dass die Worte zwischen den Pausen weniger bedeutend werden für die theatrale Darbietung als eben die Pausen. Obwohl sich in den Pausen nichts bzw. kaum akustisch Messbares tut, kann sich in einer gekonnten Pause - also einer Pause, die den soziokulturellen und künstlerischen Erfordernissen im jeweiligen Kontext genüge tut - mehr an Dramatik, Energie und Emotionen ereignen, als im klanglichen Teil einer Rede oder Geschichte.

Socio-cultural silences are related to the characteristic manner in which entire social and cultural orders refrain from speech and manipulate both psycholinguistic and interactive silences. They may define cultural patterns of communication better than words. (DANIELEWICZ 1998: 76).

Bei Hader kann eine Pause sogar eine höhere kommunikative Wichtigkeit haben, als die Geschichte, die er erzählt. Wen vom Publikum interessiert kommunikativ gesehen, dass sich auch Afrikaner ,ich liebe dich“ sagen? Was der Aussage Wichtigkeit und Eindringlichkeit verleiht, ist nicht der propositionale Gehalt, sondern die Pause ${ }^{42}$, die

\footnotetext{
${ }^{41}$ Bspw. Minute 5.30 bis 5.42 auf der DVD Im Keller oder Minute 1.44 bis 1.47 auf der DVD Privat.

${ }^{42}$ Hier mag es sich um eine bestimmte Art von Pause handeln: das Schweigen, das an dieser Stelle etwas semantisch gesehen Anakoluthisches an sich hat. Der Zuhörer wird eingeladen weiter oder fertig zu denken, wird aber in seiner romantischen Erwartungshaltung durch den nachfolgenden trockenen Kommentar Haders ernüchtert. Eine mögliche Theorie des Schweigens findet sich bspw. bei Luhmann, der es aus der Perspektive der Kommunikation betrachtet, von wo es ausgeht und deren Teil es sein kann. „Als Kommunikation in Gang gebracht, kann die Gesellschaft auch Schweigen in Kommunikation einbeziehen - etwa im Sinne des zuhörenden Schweigens, im Sinne beredten Schweigens, im Sinne des ,qui tacet consentire videtur"." (LuHMANN 1989: 17). Durch Schweigen als eine Form der Pause komme daher das Nichtsagen bzw. „daß nicht gesagt wird, was nicht gesagt wird“ (LuHMANN 1989: 16) zur Sprache, bei Hader jedenfalls auch noch das Nichtgesagte bzw. das sprachlich unmittelbar nicht Gesagte. „Und selbst diese Charakterisierung als ,Schweigen' ist noch eine der Kommunikation und noch eine mit Bezug auf Kommunikation; denn in Wirklichkeit ist ,Schweigen' ja keine Operation, die außerhalb der Gesellschaft faktisch vollzogen wird, sondern nur ein Gegenbild, das die Gesellschaft in ihre Umwelt projiziert, oder auch der Spiegel, in dem die Gesellschaft zu sehen bekommt, daß nicht gesagt wird, was nicht gesagt wird.“ (LUHMANN 1989: 16). „Mit Niklas Luhmann gilt es hier einer "Interpretation des Schweigens“ entgegenzuwirken, die lediglich ,rekursiv an das Netz [der Kommunikation] angeschlossen ' wird. Eine solche Einbindung des Schweigens in die Signifikationen des Textes hätte den Effekt, ,das Schweigen dann gar nicht mehr zu hören'.“(ELZENHEIMER 2008: 20).
}

Pandaemonium, São Paulo, v. 24, n. 44, set.-dez. 2021, p. 314-329 
GUGGENBERGER, R. - Inszenierungen und Bedeutung von Pausen in Josef Hader

Hader an dieser Stelle setzt. ${ }^{43}$ In den Pausen kann sich bei Hader konzentriertes theatrales Geschehen abspielen.

Silence is not merely an acoustic break when nothing happens. On the contrary, due to the interactive and expressive qualities of silence and pauses, communication continues, although no words are uttered (DANIELEWICZ 1998: 223).

Haders Pausen sind ${ }^{44}$ nicht gleich, sondern in ihrer Funktion extrem vielfältig: sie schaffen Spannung, lösen Betroffenheit aus, geben dem Publikum Zeit zum Reflektieren und zum Herstellen eines Bezuges des Gesagten zum jeweiligen persönlichen Verhalten und Denken. Hader:

[I]ch will wirklich unter die Haut gehen und ich will, daß das manchmal auch Grenzbereiche betreffen kann. (KÜCHLER 1995: 229; Hader im Interview). Unterhaltung hat für mich zunächst einmal eher etwas von einer Pflicht - das Pflichtprogramm im Unterschied zum Kürprogramm. Das Pflichtprogramm ist, daß die ganze Sache spannend ist, daß sie unterhaltend ist. Das andere ist, daß noch etwas anderes vorhanden sein sollte, nämlich einerseits etwas, das ich hinüberkriegen will und andererseits, das ich so hinüberkriegen will, daß es möglichst tief in einen Menschen hineingeht (KÜCHLER 1995: 224; Hader im Interview).

Die Erwartungshaltung des Zuschauers gegenüber der Performanz Haders kehrt sich in den Pausen gegen den Zuschauer selbst zurück: Er frägt sich, was von ihm in den stillen, nachdenklichen, manchmal auch vorwurfsvollen Momenten des Kabaretts erwartet wird. Diesen Effekt von Stille bzw. ,stillen Pausen“ auf das Publikum beschreibt Jensen wie folgt:

I suddenly heard all my inner voices, chattering and furious, as if they were finally able to shout out to me all the latent themes. ... Very stupid questions. What next? Why did it happen? What is the way out of this? What should be done? What did I need that for? And there is only one answer all the time: I don't know. (JENSEN 1973: 29-30; nach JAWORSKI 1993: 19).

Die „stillen Pausen“ fordern das Publikum heraus aktiv zu werden, um die Stille auszufüllen. (Cf. DANIELEWICZ 1998: 76).

Lengthy interactive silences allow time for inferences and judgements about the possible meanings of a message, including the meaning of silence. Furthermore, they facilitate judgements concerning the character, motives and personality of other speek participants. Long silence in interactive situations promotes informality and personal closeness. (DANIELEWICZ 1998: 76).

43 „Es geht hier im Unterschied zur Informationsfunktion [bei der Pausen in der Regel nur als Verständnishilfe eingesetzt werden] nicht um den kognitiven Bereich, sondern um den emotionalen. Es geht nicht darum, Unwissenheit zu verringern, Informationen zu vermitteln; es geht darum, aufzuwühlen.“ (KÜCHLER 1995: 227; Hader im Interview).

${ }^{44}$ Obgleich sie an sich und für sich selbst genommen nichts sind, sondern immer etwas brauchen, von dem sie die Unterbrechung oder die negative Alternative sind.

Pandaemonium, São Paulo, v. 24, n. 44, set.-dez. 2021, p. 314-329 
GUGGENBERGER, R. - Inszenierungen und Bedeutung von Pausen in Josef Hader

Die häufige, charakteristische Verwendung der Pausen durch Hader ist Kabarettkollegen $^{45}$ und Wissenschaftlern gleichermaßen aufgefallen. So wies etwa bereits Ringhofer auf den Effekt der persönlichen Nähe, den Hader mitunter durch die Pause zu erzeugen pflegt, hin, und widmete den Pausen bei Hader ein Unterkapitel:

Es gibt verschiedene Erklärungsmöglichkeiten, warum Hader das Stilmittel der Pause im Text so häufig verwendet. Einerseits unterstützt er damit die natürlich wirkende Sprechweise, andererseits erzeugt und erhöht er damit die Spannung, bevor die Pointe einsetzt. [...] Die Textpause (innerhalb einer Nummer oder eines Liedes), oder auch Stille ist zu einem Markenzeichen Haders geworden. Diese bewußte Unterbrechung des Redeflußes stellt eine Provokation für den Zuschauer dar. Er wird verunsichert, weil er nicht weiß, was der Kabarettist damit bewirken will. Hader setzt dieses Mittel grundsätzlich aus zwei Gründen ein. Der erste Grund, ist die schon erwähnte Natürlichkeit der Sprechweise, die Hader damit dem Zuschauer vermitteln will. Diesem soll weiß gemacht werden, daß die Privatperson Hader Intimitäten aus seinem Privatleben erzählt. [...] Im zweiten Fall handelt es sich um die Provokation einer Publikumsreaktion. Hader versucht damit Stimmungen zu erzeugen. Manche Textpassagen benötigen eine Pause, damit der Zuschauer die Worte auf sich wirken lassen kann. Dieser Effekt tritt zum Beispiel bei der Textstelle auf, in der Hader von der Todessehnsucht seiner Grußmutter erzählt. Ein halbes Jahr, nachdem sie diesen [sic! - gemeint ist der Wunsch zu sterben] aussprach verstarb sie. Genau nach diesem Satz setzt Hader eine Pause, damit kann er ein Gefühl der Betroffenheit beim Zuschauer erzeugen. Er läßt diesem keine Chance diese Bemerkung zu übergehen, der Zuschauer ist der Betroffenheit, die diese Texte erzeugen ausgeliefert. (RINGHOFER 2000: 82).

Der zweckmäßige Gebrauch und die Gestaltung der verschiedenen Arten von Pausen sind essentiell, um den „Grundberuf“ des Kabarettisten ausüben zu können: „dass man [...] verhindert, dass die Leute gehen. [...] man muss irgendwie spannend sein. Man muss die Leute in einer Spannung ${ }^{46}$ halten.“(ARENDS 2011: 112; Hader im Interview).

Hader legt großen Wert auf den dramaturgischen Aufbau, der Spannung erzeugen und somit die Konzentration fördern soll. Dabei setzt Hader zum Beispiel die Kontrastwirkung von Liedern, ruhigen Textpassagen und Gefühlsausbrüchen gegeneinander ab und trägt so zur Wachsamkeit des Publikums bei. (RINGHOFER 2000: $85)$.

An der die Wachsamkeit des Publikums bewahrenden „Kontrastarbeit“ haben auch die Pausen ihren Anteil. ${ }^{47}$ Vor allem aber ermöglichen sie „ein Gefühl der Betroffenheit im Zuschauer [zu] erzeugen“ (RINGHOFER 2000: 80), wovon die Stille im

\footnotetext{
45 „Die beiden Jungkabarettisten [...] ,Schöller \& Bacher“ erwähnten in ihrem ersten Programm ,Warten Josef Hader. Während ihres Programmes sitzen sie fast völlig unbewegt auf einer Parkbank. [...] wobei sie viele (Denk-) Pausen machen. Diese ,natürliche Sprechweise‘ verbindet sie mit Hader. [...] Schöller und Bacher weisen, nach einer Pause darauf hin, daß sie , diese Pause vom Hader haben`. Das zeugt von der Einzigartigkeit, wie und wo Hader Pausen [...] setzt.“ (RINGHOFER 2000: 81).

${ }^{46}$ „,ich hab“ selber gern als Zuschauer eine dramaturgische Spannung [Hervorhebung d. Verf.]." (ARENDS 2011: 63; Hader im Interview).

${ }^{47}$ „Wenn man sein potentielles Publikum erreicht, sind die verschiedenen Ebenen von Stille das wichtigste Barometer. [...] Manchmal geht eine bestimmte Gefühlsregung, wie eine Welle durch das Publikum, und die Art der Stille verändert sich.“(RINGHOFER 2000: 90; Hader im Interview).
}

Pandaemonium, São Paulo, v. 24, n. 44, set.-dez. 2021, p. 314-329 
GUGGENBERGER, R. - Inszenierungen und Bedeutung von Pausen in Josef Hader

Publikum, während und nachdem Hader zweimalig ,ich liebe dich“ flüstert ${ }^{48}$, beredtes Zeugnis ablegt.

Dieser altmodische Begriff Katharsis - daß man so hinaus geht, einerseits gereinigt in der Hinsicht, daß das Hirn relativ klar ist und plötzlich Zusammenhänge erkennt im Überblick über das eigene Leben oder über die Gesellschaft und andererseits auch vom Gefühl her etwas da ist, was diese Erkenntnis etwas leichter macht. Die Erkenntnis ist meistens eine traurige im Kopf. Und deswegen bin ich sehr für diese warme Brust ${ }^{49}$, mit der man hinausgehen sollte und ich tu alles dafür. (RINGHOFER 2000: 80-81; Hader im Interview).

Dass Hader an dem aristotelisch geprägten Begriff der Katharsis festhält und es andererseits unterlässt „den Unterhaltungscharakter des Theaters in Frage“ (BENJAMIN 2007: 381) zu stellen, wie es Brecht tat ${ }^{50}$, lässt vermuten, dass das epische Theater, trotz stilistischer Ähnlichkeiten mit den Kabaretts Haders, auf jene keinen direkten maßgeblichen Einfluss hat und hatte. Dass den Unterbrechungen bei Brecht einerseits ${ }^{51}$ und den Pausen bei Hader andererseits je eine wichtige Funktion zukommt, reicht zu einer anderen Bewertung - zumindest im Kontext der vorliegenden Untersuchung - nicht aus. Allerdings kann sich auch bei Hader die ,Entdeckung [...] von Zuständen [...] mittels der Unterbrechung von Abläufen“ (BENJAMIN 2007: 388) vollziehen, wie bspw. in Im Keller, wenn der Protagonist bemerkt, dass er vergessen habe, dass seine Frau ihm gesagt habe, dass sie keine Kinder von ihm möchte, weil sie durch ein Kind so stark merken würde, wie sie selbst älter würde ${ }^{52}$.

\section{Schlussbemerkung}

Nun wäre es statistisch gesehen schon höchste Zeit, dass Hader ein neues KabarettProgramm darbieten würde. Zwischen den letzten beiden eigenständigen Kabaretts ${ }^{53}$ Privat (1994) und Hader muss weg (2004) vergingen zehn Jahre, und von jenem abendfüllenden Kabarett bis heute sind es sogar schon sechzehn Jahre. Diese lange

\footnotetext{
${ }^{48}$ Minute $1 \mathrm{~h} 16.17$ bis $1 \mathrm{~h} 16.31$ auf der DVD Privat.

49 „Die Leute haben in den zwei Stunden [des Kabarettprogramms] wirklich Dinge gehört, die grauslich waren. Sie sind aber dadurch ungeheuer bereit, so eine kleine Geschichte $[\ldots]$ am Schluß, als kleinen Wärmespender oder als Lächeln oder als irgendetwas Positives, das man ihnen gibt, zu nehmen und damit noch rauszugehen" (KÜCHLER 1995: 225-226; Hader im Interview).

50 „Was in der Brechtschen Dramatik wegfiel, das war die aristotelische Katharsis, die Abfuhr der Affekte durch Einfühlung in das bewegende Geschick des Helden." (BENJAMIN 2007: 388).

51 „Für das epische Theater steht [...] die Unterbrechung der Handlung im Vordergrunde.“ (BENJAMIN 2007: 375).

${ }^{52}$ Minute 41.27 bis 41.57 auf der DVD Im Keller.

${ }^{53}$ Hader spielt Hader ist ja ein Potpourri vorangegangener Programme.
} 
GUGGENBERGER, R. - Inszenierungen und Bedeutung von Pausen in Josef Hader

Zeitspanne steht durchaus im Einklang mit dem Plot jenes bisher letzten großen kabarettistischen Streichs. Denn dort verordnete sich der Kabarettist selbst eine Generalpause, indem er sein theatrales Ego bereits nach den ersten Szenen sterben lässt. Glücklicherweise scheint es, dass sich Hader auf der Bühne doch nicht darum umbringen hat lassen, um uns künstlerisch zu sagen, dass er mit der Großform des Kabaretts abgeschlossen habe. Zumindest hat Hader in der 346sten Ausgabe der TV-Sendung Willkommen Österreich davon gesprochen, gerne wieder ein Kabarett-Programm schaffen zu wollen. 54

\section{Literaturverzeichnis}

ARENDS, Lena. Josef Hader und die Komik: anhand des Programms Hader muss weg. Diplomarbeit (Theater-, Film- und Medienwissenschaft) PhilologischKulturwissenschaftliche Fakultät, Universität Wien, Wien, 2011.

ARISTOTELES. Poetik. Arbogast Schmitt (erl.). Berlin: De Gruyter, 2008.

ARISTOTELES. Poetik. Manfred Fuhrmann (hrsg.). Stuttgart: Reclam, 1994.

BENJAMIN, Walter. Wahlverwandtschaften. Aufsätze und Reflexionen über deutschsprachige Literatur. Frankfurt am Main: Suhrkamp, 2007.

CAPPUCCINO Melange. Regie: Paul Harather. Wien: Hoanzl, 2009. DVD (92 min).

DANIELEWICZ-BetZ, Anna. Silence and Pauses in Discourse and Music. Dissertation (Applied Linguistics, Phonetics and English Studies) Philosophische Fakultät, Universität des Saarlandes, Saarbrücken, 1998.

ElZENHEIMER, Regine. „,... wenn in reicher Stille ...' Pause, Fermate und Stille im Spätwerk Luigi Nonos“. In: PrimaVesi, Patrick; MAHREnholz, Simone (hrsg.). Geteilte Zeit. Zur Kritik des Rhythmus in den Künsten. Schliengen: Argus, 2005, 71-84.

ElzenHEIMER, Regine. Pause. Schweigen. Stille. Dramaturgien der Abwesenheit im postdramatischen Musik-Theater. Würzburg: Königshausen \& Neumann, 2008.

GugGenberger, Rainer. „Der Primat des Hörens in der prähellenistischen Reflexion über die

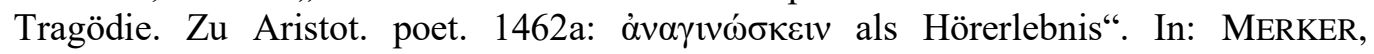
Raimund; DANEK, Georg; KLECKER, Elisabeth (hrsg.). Trilogie. Epos - Drama - Epos. Festschrift für Herbert Bannert. Wien: Praesens, 2016, 221-249.

HADER, Josef. Aschermittwoch der Kabarettisten. Youtube. Online: https://www.youtube.com/watch?v=VkFcunNnKxI\&t=40s (24/10/2017).

HADER, Josef. Im Keller. Wien: Hoanzl, 2008. DVD (55 min.) (aufgezeichnet im Orpheum Graz 1994).

HADER, Josef. Privat. Wien: Hoanzl, 2008. DVD (129 min.) (aufgezeichnet in der Burgarena Finkenstein 1996).

JAwORSKI, Adam. The Power of Silence. Social and Pragmatic Perspectives. Newbury Park, London, New Delhi: SAGE Publications, 1993.

KÜCHLER, Verena. Die zehnte Muse. Zeitgemäßes Kabarett: Form, Funktion und Wirkung einer Kommunikationsart. Dissertation (Publizistik) Fakultät für Sozialwissenschaften, Universität Wien, Wien, 1995.

\footnotetext{
${ }^{54}$ Minute 8.36 bis 8.39 in Willkommen Österreich 346-2, ausgestrahlt am 7.2.2017.
} 
GUGGENBERGER, R. - Inszenierungen und Bedeutung von Pausen in Josef Hader

LaCAN, Jacques. Das Seminar, Buch VII (1959-1960). Die Ethik der Psychoanalyse. Weinheim, Berlin: Quadriga, 1996.

LissA, Zofia. „Die ästhetischen Funktionen der Stille und Pause in der Musik“. Studien zur Musikwissenschaft 25/1962 (Festschrift für Erich Schenk). Graz, Wien, Köln: Hermann Böhlaus Nachf., 315-346, 1962.

LuHMAnN, Niklas. „Reden und Schweigen“. In: LuhmAnN, Niklas; FuchS, Peter. Reden und Schweigen. Frankfurt/Main: Suhrkamp, 1989, 7-20.

MARZULlo, Benedetto. „Die visuelle Dimension des Theaters bei Aristoteles“. Philologus 124, 189-200, 1980.

MEISTER, Monika. „Horváths Zäsuren. Dramaturgie der ,Stille““. In: MEISTER, Monika. Theater denken. Ästhetische Strategien in den szenischen Künsten. Wien: Sonderzahl, 2009, 225242.

MERKER, Raimund. „Zur verbalen Gestaltung des fiktiven Schauplatzes in der attischen Tragödie des 5. Jhdts. v. Chr.“. Caliope 31, 112-140, 2016.

RINGHOFER, Alexandra. Kabarett-Theater oder theatrale Keimzelle? Josef Haders SoloProgramm: „Privat“. Diplomarbeit (Theater-, Film- und Medienwissenschaft) Philologisch-Kulturwissenschaftliche Fakultät, Universität Wien, Wien, 2000.

WILLKOMMEN Österreich, Folge 346, ausgestrahlt am 7/2/2017, auf http://www.willkommenoesterreich.tv/pl.php?plid=346\#F346 (26/10/2017).

Recebido em 1 de fevereiro de 2021 Aceito em 28 de março de 2021 